\title{
PRIMARY OVARIAN NEOPLASMS: 5-YEAR INSTITUTIONAL STUDY
}

\author{
Nalini Modepalli', Suguna Belur Venugopal², Tirthankar Mukherjee ${ }^{3}$
}

${ }^{1}$ Assistant Professor, Department of Pathology, Rajarajeswari Medical College and Hospital, Bangalore.

2 Professor and HOD, Department of Pathology, Kempegowda Institute of Medical Sciences, Bangalore.

${ }_{3}^{3}$ Assistant Professor, Department of General Medicine, Kempegowda Institute of Medical Sciences, Bangalore.

\begin{tabular}{l}
\hline ABSTRACT \\
BACKGROUND AND OBJECTIVES \\
The wide variety of ovarian neoplasms makes it an interesting topic for study. The objectives of the present study was to study \\
clinical and histopathologic features of various ovarian tumours and classify them according to WHO classification.
\end{tabular}

\section{METHODS}

Five year study was conducted on ovarian specimens received in Central Laboratory, Department of Pathology, Kempegowda Institute of Medical Sciences, Bangalore, from June 2006 to May 2011. All ovarian specimens which on histopathological examination was diagnosed as primary ovarian neoplasm was included in the study.

\section{RESULTS}

We studied 210 primary ovarian tumours of ovary of which $83.97 \%$ were benign tumours, $3.77 \%$ were borderline epithelial and $12.26 \%$ were malignant tumours. Surface epithelial tumours were more common followed by Germ cell tumours. Mean age of diagnosis was 40.43 years. Abdominal mass was the most common presenting symptom. Right sided tumours were more common than the left.

\section{CONCLUSION}

Even though immunohistochemical and chromosomal studies have made diagnosis and differentiation of tumours easier, in developing countries like India, cost effective histomorphologic studies still form the backbone of diagnosis of these tumours.

\section{KEYWORDS}

Primary Ovarian Neoplasms, Surface Epithelial Tumours, Germ Cell Tumours, Sex Cord Stromal Tumours.

HOW TO CITE THIS ARTICLE: Modepalli N, Venugopal SB, Mukherjee T. Primary ovarian neoplasms: 5-year institutional study. J. Evolution Med. Dent. Sci. 2016;5(46):2904-2908, DOI: 10.14260/jemds/2016/677

\section{INTRODUCTION}

Ovary is a small organ, unique in terms of variety of lesions that arise from it. ${ }^{1,2}$ Ovarian cancer is $6^{\text {th }}$ most common cancer among women worldwide, ${ }^{3,4} 3^{\text {rd }}$ leading site in India. ${ }^{5}$ Tumours of the ovary are amazingly diverse pathological entities, attributable to 3 cell types that make up normal ovary-multipotent surface (Coelomic) covering epithelium, sex cord stromal cells and totipotent germ cells. Ovarian cancer has the worst prognosis among gynaecological malignancies, 5-year survival rate being $45 \% .4,6$ It is generally impossible to diagnose nature of ovarian tumours preoperatively. Histopathologic examination is needed for a confirmatory diagnosis. ${ }^{7}$ Knowing the type of tumour helps in judicious management of the patient.

\section{MATERIALS AND METHODS}

Five year study was conducted on all ovarian specimens received in the Central Laboratory, Department of Pathology, Kempegowda Institute of Medical Sciences (KIMS), Bangalore, which on histopathological examination were diagnosed as tumour listed under WHO classification of primary ovarian

Financial or Other, Competing Interest: None.

Submission 11-03-2016, Peer Review 19-05-2016,

Acceptance 25-05-2016, Published 09-06-2016.

Corresponding Author:

Dr. Nalini Modepalli,

Flat No. 104, $2^{\text {nd }}$ Floor,

Shravanthi Orchids, 1st Main,

Revenue Layout, Padmanabhanagar,

Bangalore-560070

E-mail: doctor.nalini@gmail.com

DOI: $10.14260 /$ jemds/2016/677 tumours. ${ }^{3}$ Non-neoplastic lesions, tumour-like conditions, paraovarian lesions and metastatic tumours were excluded from the study.

Specimens were sent in $10 \%$ formalin. Weight, measurement, external appearance, appearance of capsule, features on cut section and other relevant features recorded. Representative sampling was ensured for accurate examination.

Material was processed routinely. Multiple thin sections of 4-5 micron thickness were obtained from paraffin blocks, stained with $\mathrm{H}$ and $\mathrm{E}$ for microscopic examination.

Clinical history was noted. Special stains and immunohistochemistry was done when necessary. All tumours were classified as per WHO classification.

\section{RESULTS AND OBSERVATIONS}

Our study included 210 cases (212 specimens) of primary ovarian neoplasms; $66.2 \%$ - Surface Epithelial Stromal (SES) tumours, 58 (27.6\%) - Germ Cell (GC) tumours and 13 (6.2\%) - Sexcord Stromal (SS) tumours (Table 1).

Two cases of bilateral SES tumours had 2 different diagnoses on either side and hence we have 212 diagnoses from 210 cases (141 diagnoses from 139 cases of SES tumours); $83.97 \%$ were benign, $12.26 \%$ malignant and $3.77 \%$ were borderline epithelial tumours. Of benign tumours, $65.2 \%$ cases SES, 29.2\% GC and 5.6\% were SS tumours. Among malignant tumours, $65.14 \%$ SES, $23 \%$ GC and $11.6 \%$ SS tumours reported.

Mean age at presentation was 40.43 yrs. GC tumours were seen in younger age group while SS tumours were in the older. (Table 2). 
Mass abdomen was most common presenting symptom. Menstrual disturbances were more common in SS tumours. (Table 3).

197 (93.8\%) of primary ovarian neoplasms were U/L and $13(6.2 \%)$ B/L. All bilateral cases were SES tumours.

$132(59.2 \%)$ cases were right sided and 91 (40.8\%) left sided.

Size of tumours ranged from $2 \times 2 \times 1 \mathrm{~cm}$ (Mature cystic teratoma) to $43 \times 30 \times 15 \mathrm{~cm}$ (Mucinous cystadenoma).

Benign tumours showed $51.8 \%$ unilocularity and $48.2 \%$ multilocularity. Malignant tumours were more commonly multilocular (64.7\%).

$154(72.6 \%)$ cases were purely cystic, $16(7.5 \%)$ solid and $42(19.8 \%)$ partly cystic and partly solid areas; $84.8 \%$ of benign tumours were purely cystic, 5.6\% solid and $9.6 \%$ partly cystic and partly solid. Among malignant tumours, 20 (76.9\%) were of mixed consistency and $6(23.1 \%)$ purely solid. None of the malignant tumours were purely cystic.

\begin{tabular}{|c|c|c|}
\hline Tumour Type & Frequency & Percent \\
\hline SES & 139 & 66.2 \\
\hline GC & 58 & 27.6 \\
\hline SS & 13 & 6.2 \\
\hline Total & $\mathbf{2 1 0}$ & $\mathbf{1 0 0 . 0}$ \\
\hline \multicolumn{2}{|c|}{ Table 1: Frequency of Primary Ovarian Neoplasms } \\
\hline
\end{tabular}

\begin{tabular}{|c|c|c|c|c|c|c|c|c|}
\hline \multirow{2}{*}{ SES } & \multicolumn{7}{|c|}{ Age (yrs.) } & \multirow{2}{*}{ Total } \\
\cline { 2 - 10 } & $<\mathbf{2 0}$ & $\mathbf{2 0 - 2 9}$ & $\mathbf{3 0 - 3 9}$ & $\mathbf{4 0 - 4 9}$ & $\mathbf{5 0 - 5 9}$ & $\mathbf{6 0 - 6 9}$ & $\mathbf{2 7 0}$ & \\
\cline { 2 - 10 } & $2.9 \%$ & $15.8 \%$ & $18.0 \%$ & $\mathbf{3 5 . 3 \%}$ & $15.1 \%$ & $6.5 \%$ & $6.5 \%$ & $100.0 \%$ \\
\hline \multirow{2}{*}{ GC } & 6 & 22 & 11 & $\mathbf{1 4}$ & 2 & 3 & 0 & 58 \\
\cline { 2 - 9 } & $10.3 \%$ & $37.9 \%$ & $19.0 \%$ & $\mathbf{2 4 . 1 \%}$ & $3.4 \%$ & $5.2 \%$ & $.0 \%$ & $100.0 \%$ \\
\hline \multirow{2}{*}{ SS } & 0 & 0 & 4 & $\mathbf{2}$ & 1 & 6 & 0 & 13 \\
\cline { 2 - 9 } & $.0 \%$ & $.0 \%$ & $30.8 \%$ & $\mathbf{1 5 . 4 \%}$ & $7.7 \%$ & $46.2 \%$ & $.0 \%$ & $100.0 \%$ \\
\hline \multirow{2}{*}{ Total } & 10 & 44 & 40 & $\mathbf{6 5}$ & 24 & 18 & 9 & 210 \\
\cline { 2 - 8 } & $\mathbf{4 . 8 \%}$ & $\mathbf{2 1 . 0 \%}$ & $\mathbf{1 9 . 0 \%}$ & $\mathbf{3 1 . 0 \%}$ & $\mathbf{1 1 . 4 \%}$ & $\mathbf{8 . 6 \%}$ & $\mathbf{4 . 3 \%}$ & $\mathbf{1 0 0 . 0 \%}$ \\
\hline \multicolumn{7}{|c|}{ Table 2: Age Distribution } \\
\hline
\end{tabular}

\begin{tabular}{|c|c|c|c|c|}
\hline Sass Abdomen & $\begin{array}{c}58.9 \% \\
(83)\end{array}$ & $\begin{array}{c}39.7 \% \\
(23)\end{array}$ & $\begin{array}{c}38.5 \% \\
(5)\end{array}$ & $\begin{array}{c}52.4 \% \\
(111)\end{array}$ \\
\hline Pain Abdomen & $\begin{array}{c}35.5 \% \\
(50)\end{array}$ & $\begin{array}{c}29.3 \% \\
(17)\end{array}$ & $\begin{array}{c}23.1 \% \\
(3)\end{array}$ & $\begin{array}{c}33 \% \\
(70)\end{array}$ \\
\hline $\begin{array}{c}\text { Menstrual } \\
\text { Disturbances }\end{array}$ & $\begin{array}{c}13.5 \% \\
(19)\end{array}$ & $\begin{array}{c}13.8 \% \\
(8)\end{array}$ & $\begin{array}{c}53.8 \% \\
(7)\end{array}$ & $\begin{array}{c}16 \% \\
(34)\end{array}$ \\
\hline Ascitis & $\begin{array}{c}14.9 \% \\
(21)\end{array}$ & $\begin{array}{c}6.9 \% \\
(4)\end{array}$ & $\begin{array}{c}7.7 \% \\
(1)\end{array}$ & $\begin{array}{c}12.3 \% \\
(26)\end{array}$ \\
\hline Urinary & $\begin{array}{c}1.4 \% \\
(2)\end{array}$ & $0 \%$ & $0 \%$ & $\begin{array}{c}0.9 \% \\
(2)\end{array}$ \\
\hline Infertility & $\begin{array}{c}1.4 \% \\
(2)\end{array}$ & $\begin{array}{c}3.4 \% \\
(2)\end{array}$ & $0 \%$ & $\begin{array}{c}1.9 \% \\
(4)\end{array}$ \\
\hline GIT Symptoms & $\begin{array}{c}2.8 \% \\
(4)\end{array}$ & $0 \%$ & $0 \%$ & $\begin{array}{c}1.9 \% \\
(4)\end{array}$ \\
\hline Asymptomatic & $\begin{array}{c}11.3 \% \\
(16)\end{array}$ & $\begin{array}{c}19 \% \\
(11)\end{array}$ & $0 \%$ & $\begin{array}{c}12.7 \% \\
(27)\end{array}$ \\
\hline \multicolumn{5}{|c|}{ Table 3: Clinical Presentation } \\
\hline \multicolumn{5}{|c|}{}
\end{tabular}

\begin{tabular}{|c|c|c|c|}
\hline $\begin{array}{l}\text { Sl. } \\
\text { No. }\end{array}$ & Tumour & Number & Percentage \\
\hline \multicolumn{4}{|c|}{ Surface Epithelial Stromal Tumours } \\
\hline \multicolumn{4}{|c|}{ Serous Tumours } \\
\hline 1 & Serous Cystadenoma & 53 & $25 \%$ \\
\hline 2 & $\begin{array}{l}\text { Papillary Serous } \\
\text { Cystadenoma }\end{array}$ & 8 & $3.8 \%$ \\
\hline 3 & Serous Cystadenofibroma & 6 & $2.8 \%$ \\
\hline 4 & Adenofibroma & 2 & $0.9 \%$ \\
\hline 5 & $\begin{array}{l}\text { Papillary Serous } \\
\text { Cystadenofibroma }\end{array}$ & 2 & $0.9 \%$ \\
\hline 6 & $\begin{array}{l}\text { Borderline Serous } \\
\text { Tumour }\end{array}$ & 1 & $0.5 \%$ \\
\hline 7 & $\begin{array}{l}\text { Borderline Serous } \\
\text { Cystadenofibroma }\end{array}$ & 1 & $0.5 \%$ \\
\hline 8 & $\begin{array}{l}\text { Borderline Papillary } \\
\text { Serous Tumour }\end{array}$ & 1 & $0.5 \%$ \\
\hline 9 & $\begin{array}{c}\text { Serous } \\
\text { Cystadenocarcinoma }\end{array}$ & 5 & $2.4 \%$ \\
\hline 10 & $\begin{array}{c}\text { Serous } \\
\text { Cystadenocarcinoma- } \\
\text { Solid Type } \\
\end{array}$ & 1 & $0.5 \%$ \\
\hline 11 & $\begin{array}{c}\text { Papillary Serous } \\
\text { Cystadenocarcinoma } \\
\end{array}$ & 7 & $3.3 \%$ \\
\hline \multicolumn{4}{|c|}{ Mucinous Tumours } \\
\hline 12 & Mucinous Cystadenoma & 39 & $18.4 \%$ \\
\hline 13 & $\begin{array}{c}\text { Mucinous } \\
\text { Cystadenofibroma }\end{array}$ & 1 & $0.5 \%$ \\
\hline 14 & $\begin{array}{l}\text { Borderline Mucinous } \\
\text { Tumour }\end{array}$ & 2 & $0.9 \%$ \\
\hline 15 & $\begin{array}{c}\text { Borderline Mucinous } \\
\text { Tumour with } \\
\text { Intraepithelial Carcinoma }\end{array}$ & 1 & $0.5 \%$ \\
\hline 16 & $\begin{array}{c}\text { Borderline Mucinous } \\
\text { Tumour with } \\
\text { Microinvasion } \\
\end{array}$ & 1 & $0.5 \%$ \\
\hline 17 & $\begin{array}{c}\text { Mucinous } \\
\text { Cystadenocarcinoma }\end{array}$ & 2 & $0.9 \%$ \\
\hline 18 & $\begin{array}{l}\text { Seromucinous } \\
\text { Cystadenoma }\end{array}$ & 2 & $0.9 \%$ \\
\hline 19 & $\begin{array}{c}\text { Borderline Seromucinous } \\
\text { Tumour }\end{array}$ & 1 & $0.5 \%$ \\
\hline \multicolumn{4}{|c|}{ Clear Cell Tumours } \\
\hline 20 & Clear cell carcinoma & 1 & $0.5 \%$ \\
\hline \multicolumn{4}{|c|}{ Endometrioid Tumours } \\
\hline 21 & $\begin{array}{c}\text { Endometrioid } \\
\text { Adenocarcinoma }\end{array}$ & 1 & $0.5 \%$ \\
\hline \multicolumn{4}{|c|}{ Brenner Tumours } \\
\hline 22 & Benign Brenner Tumour & 3 & $1.4 \%$ \\
\hline \multicolumn{4}{|c|}{ Sex Cord Stromal Tumours } \\
\hline 23 & Granulosa Cell Tumour & 7 & $3.3 \%$ \\
\hline 24 & $\begin{array}{c}\text { Granulosa-Theca Cell } \\
\text { Tumour }\end{array}$ & 1 & $0.5 \%$ \\
\hline 25 & Luteinised Thecoma & 1 & $0.5 \%$ \\
\hline 26 & Fibroma & 2 & $0.9 \%$ \\
\hline 27 & $\begin{array}{l}\text { Fibroma with Minor } \\
\text { Sexcord Elements }\end{array}$ & 1 & $0.5 \%$ \\
\hline 28 & Stromal Luteoma & 1 & $0.5 \%$ \\
\hline \multicolumn{4}{|c|}{ Germ Cell Tumours } \\
\hline 29 & Immature Teratoma & 1 & $0.5 \%$ \\
\hline 30 & Mature Teratoma & 52 & $24.5 \%$ \\
\hline 31 & Dysgerminoma & 4 & $1.9 \%$ \\
\hline \multirow[t]{2}{*}{32} & $\begin{array}{l}\text { Malignant Mixed Germ } \\
\text { Cell Tumour }\end{array}$ & 1 & $0.5 \%$ \\
\hline & Total & 212 & $100 \%$ \\
\hline & e 4: Distribution of Prin & Ovaric & oplasms \\
\hline
\end{tabular}




\begin{tabular}{|c|c|c|c|}
\hline & Benign & Malignant & Total \\
\hline Seromucinous & 2 & 1 & 3 \\
\hline Endometrioid & & 1 & 1 \\
\hline Transitional & 3 & $\begin{array}{c}\text { 1(PAS stain } \\
\text { demonstrating } \\
\text { hyaline } \\
\text { globules) }\end{array}$ & 1 \\
\hline Mixed epithelial & $\begin{array}{c}\text { Left ovary-serous } \\
\text { cystadenofibroma } \\
\text { with minor Brenner } \\
\text { component (20\%) }\end{array}$ & 1 \\
\hline \multicolumn{3}{|c|}{ Table 5: Other SES Tumours } \\
\hline
\end{tabular}

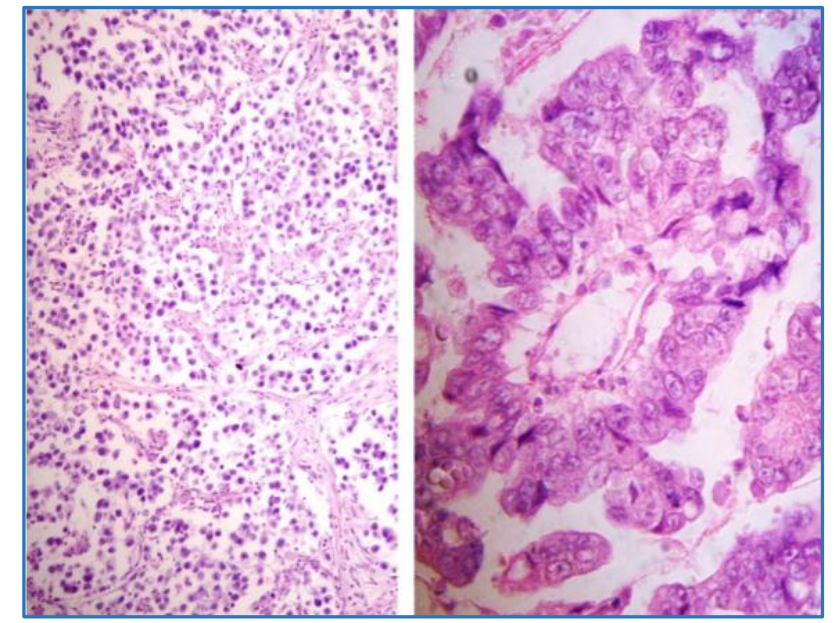

Fig. 5A \& B: Malignant Mixed Germ Cell Tumour (Dysgerminoma and Yolk Sac Components)

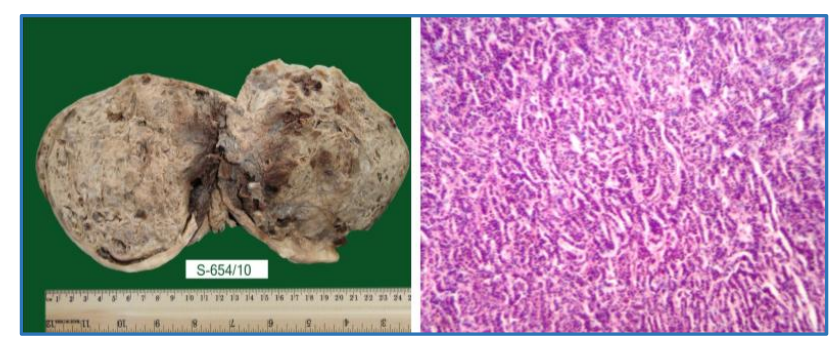

Fig. 6: Granulosa Cell Tumour

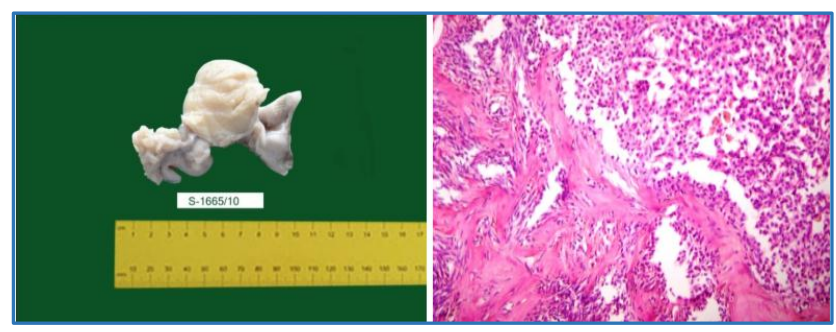

Fig. 7: Luteinised Thecoma

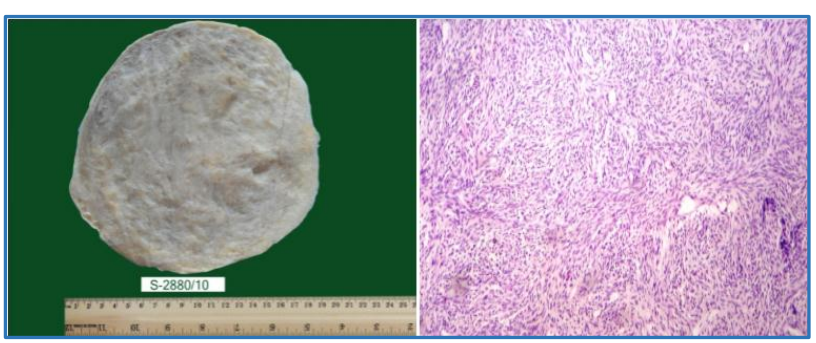

Fig. 8: Fibroma

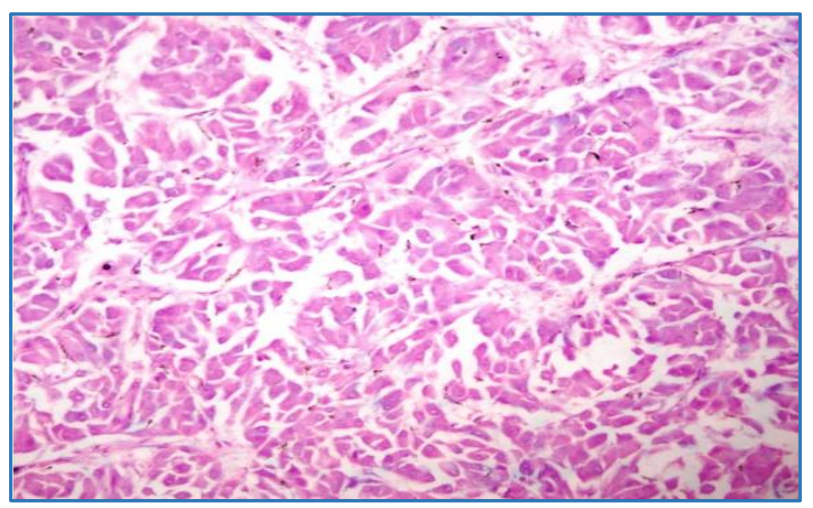

Fig. 9: Stromal Luteoma

Fig. 4: Dysgerminoma

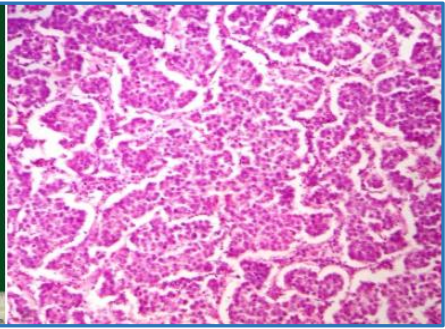

simping 


\section{DISCUSSION}

Primary Ovarian Neoplasms Are Broadly classified into

1. Surface Epithelial-Stromal Tumours (SES).

2. Germ Cell Tumours (GC).

3. Sex Cord Stromal Tumours (SS).

In our study, $66.2 \%$ of cases were SES, $27.6 \%$ GC and $6.2 \%$ SS tumours (Table 4), distribution comparable with studies done by Swamy et $\mathrm{al}^{8}$, Gupta et $\mathrm{al}^{2}$ and Pilli et al. ${ }^{9}$

Incidence of malignant tumours was less compared to other studies, probably because study was undertaken in a general hospital and malignant tumours diagnosed before surgery get referred to speciality oncology centres.

Ovarian tumours can occur at any age. In our study, youngest patient was 14 year old, oldest 76 years old, comparable with studies of Swamy et al ${ }^{8}$, Kayasta $\mathrm{S}^{10}$ and Kar T et al. ${ }^{11}$

Mass abdomen was most common presenting symptom followed by pain abdomen, comparable with findings of Gupta SC et $\mathrm{al}^{12}$, Bhuvanesh and Logambal ${ }^{13}$ and Jagadeeswari et al ${ }^{14}$, Pilli GS et al ${ }^{9}$, Couto $\mathrm{F}$ et $\mathrm{al}^{15}$ and Randhawa I et al ${ }^{16}$ Menstrual symptoms noted in $16 \%$ of the cases, $>50 \%$ of the patients had SS tumours (Functional tumours).

93.8\% were unilateral, $6.2 \%$ were bilateral consistent with findings of Couto F et al ${ }^{15}$, Misra RK et al ${ }^{17}$ and Prabakar BR et al. ${ }^{18}$

Right-sided tumours were more common than left, comparable with Ramachandran et $\mathrm{al}^{19}$, Pilli GS et $\mathrm{al}^{9}$ and Saxena HMK et al.20

\section{SURFACE EPITHELIAL TUMOURS}

\section{Serous Tumours}

Benign serous tumours - 50.8\%, borderline - $2.25 \%$ and malignant - $8 \%$ of all the SES tumours consistent with findings of Madan et al ${ }^{1}$ and Maheshwari et al. ${ }^{21}$

\section{Mucinous Tumours}

Benign mucinous tumours - 29.5\%, borderline - 3\% and malignant - $1.5 \%$, of all SES tumours comparable to the findings of Couto $\mathrm{F}$ et al ${ }^{15}$ and Madan $\mathrm{A}$ et al. ${ }^{1} \mathrm{IHC}$ was done for both cases of Mucinous cystadenocarcinoma, showed CK 7 positivity and CK 20 Negative, confirming that they were primary ovarian neoplasms.

Other SES tumours-described in Table 5.

One case of Synchronous Tumour of Ovary, Fallopian Tube and Cervix, comprising primary papillary cystadenocarcinoma of ovary and fallopian tube and adenosquamous carcinoma of the cervix. Synchronous multiple tumours of female genital tract are relatively rare comprising only $1-6 \%$ of genital neoplasms. ${ }^{22}$ Cases of triple synchronous primaries are extremely rare and all cases reported till date involving cervix, endometrium and ovary. ${ }^{23}$ To the best of our knowledge, this is the first case of synchronous cervical, fallopian tube and ovarian carcinomas.

2 B/L SES tumours with different diagnosis were reported. One had serous cystadenofibroma on the right side and serous cystadenoma on the left. Another had papillary serous cystadenoma on the right and borderline serous tumour on left side.

\section{Morphology of SES Tumours}

- Mucinous tumours were comparatively larger than serous.
- $\quad 90.6 \%$ were U/L and $9.4 \%$ B/L.

- $\quad 75.9 \%$-cystic, $2.8 \%$-solid and $21.3 \%$-partly cystic and partly solid.

- $57.9 \%$ had smooth external surface, $35.7 \%$ nodular/bosselated; $4.2 \%$ showed surface papillary projections.

- All benign tumours had intact capsule; $4.3 \%$ showed breached capsule (All were malignant); 2.1\% malignant tumours showed adhesions.

- $50.4 \%$ showed multilocularity, $46.8 \%$ were unilocular.

- Serous tumours were more commonly unilocular (66.7\%)

- $84.8 \%$ of mucinous tumours-multilocular, $15.2 \%$ unilocular.

- $\quad 25.5 \%$ showed papillary excrescences on cut section.

- Secondary changes in the form of haemorrhage, calcification, necrosis were more common in malignant tumours (84.6\%).

\section{Germ Cell Tumours}

Of the 210 cases $27.6 \%$ were germ cell tumours, comprising of $24.76 \%$ mature cystic teratomas (2 cases of struma ovarii, 1 strumal carcinoid and 1 squamous cell carcinoma arising in mature cystic teratoma reported), one case of immature teratoma with gliomatosis peritonei, 4 cases of malignant dysgerminoma and one malignant mixed germ cell tumour (dysgerminoma+yolk sac component). Findings were comparable to finding of Chhanda et $\mathrm{al}^{24}$ and Sahu et al.25 There are a very few cases reported in English literature on squamous cell carcinomas arising in dermoid cyst. ${ }^{26}$

Age range of mature cystic teratoma was 16-65 years, comparable to findings of Couto $\mathrm{F}$ et al ${ }^{15}$ and Pilli GS et al. ${ }^{9}$ Dysgerminomas was noted between 17-23 years comparable to findings of Gault EW et al. ${ }^{27}$

\section{Morphology of GC Tumours}

- $\quad$ All GC tumours were U/L.

- External surface - smooth in $62.11 \%$, nodular in others.

- Capsule was intact in $91.4 \%$, breached in rest of the cases. Breached capsule was seen in malignant tumours.

- $55.6 \%$ of tumours-unilocular, $44.4 \%$ - multilocular.

- Smallest was mature cystic teratoma and largest GC tumour was immature teratoma (35 cm diameter).

\section{Sex Cord Stromal Tumours}

13 sex cord stromal tumours were diagnosed. Meigs' syndrome was seen in 2 patients with ovarian fibroma. The age range was $30-65$ years; $50 \%$ of patients presented with menstrual irregularities.

\section{Morphology of SS Tumours}

- $\quad$ All the SS tumours were U/L.

- $66.7 \%$ had a smooth external surface, $33.3 \%$ were nodular.

- Capsule was intact in all except 2 malignant tumours.

- All tumours were solid, 50\% soft and 50\% firm in consistency.

- Silver impregnation for reticulin fibres was done for Granulosa and theca cell tumours. Granulosa cell tumours showed reticulin fibres surrounding aggregates of granulosa cells, whereas reticulin fibres typically surrounded individual tumour cells in theca cell tumours. 
- Van Gieson stain was done for fibromas to highlight abundant dense collagen secreted by tumour cells.

\section{Complications}

Torsion noted in $6.1 \%$ cases. Nonspecific inflammatory changes in 5.6\% cases, comparable with findings of Bhuvanesh \& Logambal. ${ }^{13}$ Two cases of SES tumours were associated with endometriosis.

\section{Associated Findings in Cervix}

Of the 55 hysterectomy specimens available, one case was of synchronous adenosquamous carcinoma of the cervix with primary papillary cystadenocarcinoma of ovary and fallopian tube. Another case was large non-keratinizing squamous cell carcinoma of cervix associated with serous cystadenoma of ovary; $91 \%$ - chronic cervicitis, $5.4 \%$ procidential changes and $3.6 \%$ malignancy noted.

\section{Associated Findings in Fallopian Tube}

33 fallopian tubes were unremarkable. One case was part of synchronous primary malignancies (Described above); $45.5 \%$ hematosalpinx, 9\% hydrosalpinx and 13.6\% non-specific salpingitis reported. One case had endometriosis and one case of ovarian adenofibroma showed tubercular salpingitis.

\section{Associated Findings in Uterus}

$29.09 \%$ proliferative, $9 \%$ secretory, $21.8 \%$ atrophic and $18.8 \%$ non-secretory endometrium noted; $11 \%$ endometrial hyperplasia and $3.6 \%$ cases with endometrial polyp seen. $23.63 \%$ cases had leiomyoma and $36.3 \%$ adenomyosis.

\section{REFERENCES}

1. Madan A, Tyagi SP, Mohsin S. Incidence of ovarian tumours at aligarh with particular reference to histopathologic typing. J Obst Gynaec Ind 1978;827-32.

2. Gupta N, Bisht D, Agarwal AK, et al. Retrospective and prospective study of ovarian tumours and tumour-like lesions. Indian J Pathol Microbiol 2007;50(3):525-7.

3. Parkin DM, Bray F, Ferlay J, et al. Global cancer statistics, 2002. CA Cancer J Clin 2005;55(2):74-108.

4. Basu P, De P, Mandal S, et al. Study of pattern of care of ovarian cancer patients in specialized cancer institute in Kolkata, eastern India. Ind J Cancer 2009;46(1):28-33.

5. Consolidated report of population based cancer registries 2001-2004. National cancer registry program. Indian council of medical research. Bangalore, 2006.

6. Jemal A, Siegel R, Ward E, et al. Cancer statistics, 2006. CA Cancer J Clin 2006;56(2):106-30.

7. Bhattacharya MM, Shinde SD, Purandare VN. A clinicpathological analysis of 270 ovarian tumours. J Postgrad Med 1980;26(2):103-7.

8. Swamy GG, Satyanarayana N. Clinicopathological analysis of ovarian tumours: a study on five years samples. Nepal Med Coll J 2010;12(4):221-3.
9. Ganga S Pilli, Sunitha KP, Dhaded AV, et al. Ovarian tumours a study of 282 cases. J Indian Med Associ 2002;100(7):420-4.

10. Kayastha S. Study of ovarian tumours in Nepal medical college teaching hospital. Nepal Med Coll J 2009;11(3):200-2.

11. Kar Tushar, Kar Asanranthi, Mohapatra PC. Intraoperative cytology of ovarian tumours. J Obstet Gynaecol India 2005;55(4):345-9.

12. Gupta SC, Singh PA, Mehrotra TN, et al. A clinico pathological study of ovarian tumours. Indian J Pathol Microbiol 1986;29(4):354-62.

13. Bhuvanesh U, Logambal A. Study of ovarian tumours. J Obstet Gynaec Ind 1978;28:271-7.

14. Jagadeshwari N, Reddy R, Rao KS. Germ cell tumours of the ovary with special study of dysgerminoma. J Obstet Gynaecol India 1972;22:366-71.

15. Couto F, Nadkarni NS, Jose M. Ovarian tumours in Goa: a clinicopathological study. J Obst Gynaec 1993;40(2):40811.

16. Randhawa I, Lata P. A study of ovarian neoplasms. J Obstet Gynaecol India 1980;30:531-6.

17. Mishra RK, Sharma SP, Gupta U, et al. Pattern of ovarian neoplasms in eastern UP. J Obstet Gynaecol India 1991;41(2):242-6.

18. Prabhakar BR, Kalyani Mangi. Ovarian tumoursprevalence in Punjab. Indian J Pathol Microbiol 1989;32(4):276-81.

19. Ramachandra G, Harilal KR, Chinnamma KK, et al. Ovarian neoplasms: a study of 903 cases. J Obstet Gynaecol India 1972;22:309-315.

20. Saxena KM, Devi G, Prakash P. Ovarian neoplasms: a retrospective study of 356 cases. J Obst Gynaec Ind 1980;20(6):522-7.

21. Maheshwari V, Tyagi SP, Sexena K, et al. Surface epithelial tumours of the ovary. Indian J Pathol Microbiol 1994;37(1):75-85.

22. Seo-Yun Tong, Yong-Sek Lee. Clinical analysis of synchronous neoplasms of female reproductive tract. Eur J Obstet Gynaec Reprod Biol 2008;136(1):78-82.

23. Hale CS, Lee L, Mittal K. Triple synchronous primary gynaecologic carcinomas: a case report and review of literature. Int Jrnl Surg Pathol 2011;19(4):552-5.

24. Chhanda M, Mishra SD. Ovarian tumours a ten year study. J Obstet Gynaecol India 1991;41(5):691-6.

25. Sahu L, Mallik RL, Nanda M. Germ cell tumours of the ovary: review of 46 cases. J Obst Gynaec 1990;40(3):446450.

26. Prasad S, Suguna BV, Ravindra S. Bilateral ovarian squamous cell carcinoma with an with an antecedent dermoid cyst in the left ovary. J Obstet Gynaecol Res 2011;37(9):1238-40.

27. Gault EW, Balsubramanyan M, Thomas E. Ovarian tumours: an analysis of 317 consecutive ovarian tumours. Ind J Med Sc 1954;8:522-535. 